\title{
GREEN CHEMISTRY TEACHING FOR SUSTAINABILITY IN PAPERS PUBLISHED BY THE JOURNAL OF CHEMICAL EDUCATION
}

\author{
Carlos Alberto Marques*a, ${ }^{*, a}$, Leonardo V. Marcelino ${ }^{a}$, Érica D. S. Dias ${ }^{b}$, Patricia Link Rüntzel ${ }^{\text {b }}$, Leila Cristina Aoyama \\ Barbosa Souza $^{c}$ and Adélio Machado ${ }^{d}$

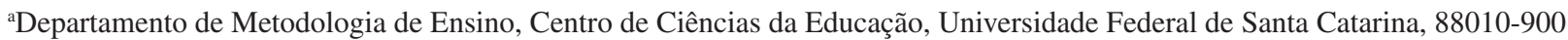 \\ Florianópolis - SC, Brasil

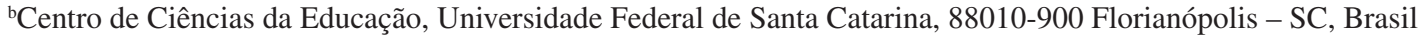 \\ 'Escola Técnica Estadual de Educação Profissional e Tecnológica de Rondonópolis, 78735-655 Rondonópolis - MT, Brasil \\ dDepartamento de Química e Bioquímica, Faculdade de Ciências, Universidade do Porto, Portugal
}

Recebido em 01/04/2020; aceito em 24/06/2020; publicado na web em 13/08/2020

\begin{abstract}
This paper discusses 286 proposals and reports of Green Chemistry (GC) teaching experiences, in papers of the Journal of Chemical Education (JCEd) until 2019. The analysis was based on previous categories: source-problem, paper focus, subjects/area of knowledge, target groups, GC contents, type of approach and purpose(s) of the proposal. A list of possible characteristics of each category served as an example to compare with the information resulting from the analyses, and thus improve the discussions. In 127 papers, GC and its teaching were associated, albeit generally, with the theme of sustainability/sustainable development, which points to its potential to face environment-related aspects of chemistry in teaching. A systemic vision in the interconnection between GC and sustainability/sustainable development appeared more explicitly in 39 papers up to 2019. So far, the analysis highlights a certain reproduction of traditional chemistry teaching, and little evidence of the particularities of GC teaching. Despite the intention of inserting GC into chemistry teaching, proposals for its incorporation are often made superficially, more as an addendum or an extra quality than a specific goal. More methodological detail of the experiments carried out is needed to help in the dissemination of GC in the training curriculum of chemists. The discussions and reports on teaching approaches based on Systems Thinking showed the opening of a promising new methodological challenge within this theoretical paradigm.
\end{abstract}

Keywords: green chemistry education; sustainability education; systems thinking.

\section{INTRODUCTION}

Chemists' endorsement of the so-called Green Chemistry (GC) can be interpreted as an expression of commitment to cleaner chemical practices, increasingly being associated with Sustainable Development (SD) efforts. Their output is comprised of research aimed at developing more benign and safe processes and products, using renewable instead of fossil-based resources whenever possible, with a view to their application in industrial situations. These features are highlighted in the Twelve Principles of GC (12P), which essentially seek to encourage innovative practices that allow cleaner and more harmless chemistry for human and environmental health.

However, the nature of these studies requires not only technical training, but also a rationality that goes beyond it, involving theoretical and practical rationality, and incorporating socio-environmental and ethical values into science. As Leff ${ }^{1}$ (p. 207) states, the environmental crisis is the first real-world crisis produced by ignorance of knowledge, world conception and control over nature. Finally, it is necessary to ask whether in the field of Environmental Sciences the conflict between technology and natural systems remains. For Ortega y Gasset, ${ }^{2}$ technology is the reform of nature (p. 14), thus it is the opposite of the adaptation of the subject to the medium, since it is the adaptation of the medium to the subject (idem, p. 17). Therefore, human beings do not adapt themselves; they rather adapt the environment (which may include other human beings) to their purposes, expressing their anthropocentric stance.

At the time of its emergence, in the early 1990s, GC was located within the framework of Pollution Prevention (P2), encouraged by

\footnotetext{
*e-mail: carlos.marques@ufsc.br
}

the Pollution Prevention Act. This catalyzed attitudinal changes from an environmentally insensitive chemistry to a "new" chemistry proactively committed to the environmental quality. Formed in the ideal of Pollution Prevention and Benign by Design Chemistry, academic output has been profiling and identifying the field of GC linked to its $12 \mathrm{P}$, formulated in 1998, in the form of a list of individualized and incremental prescriptions, ${ }^{3}$ resulting in, almost invariably, that the $12 \mathrm{P}$ were cited and used individually. It took nearly ten years for its proponents to advance the idea that the $12 \mathrm{P}$ should be taken together as a cohesive system, ${ }^{4}$ and they did so without detailing what this would mean in practice, for example, as regards the interactions between principles; they also did not mention the change to the systemic posture that the cohesive management of the $12 \mathrm{P}$ requires. This change is fundamental so that the use of $12 \mathrm{P}$ in GC supports safe increases of chemical greenness in all its dimensions and can face the issue of sustainability in face of the complexity of chemistry. Perhaps for this reason, the connection of GC with the dimensions and purposes of sustainability/SD was only diffusely highlighted in the dissemination of research and events that marked its initial phase, not deserving further discussion by its main precursors.

In just over 25 years, GC has grown and diversified, by means of a vast scientific output that claimed GC status and denomination. However, this increase contrasts with a slower growing output focused on GC's curriculum and teaching experiences. A search in the Web of Science database (Core Collection, all indexes) realized in March 2020 for the term green chemistry and its derivatives ("green* chem*") in titles, abstracts and keywords retrieved 10,540 documents (articles, reviews and editorial materials) published between 1990 and 2018. The same search using the term green chemistry together with education, learning, teaching and its radical derivatives (TS=("green* 
chem*") AND TS=(educ* OR learn* OR teach*)) retrieved only 344 articles, reviews or editorial materials. Those data corroborate how inceptive is the green chemistry education research if compared to green chemistry itself.

That search on the Web of Science also shows that almost half of the scientific outcome on green chemistry education is published in the Journal of Chemical Education (JCEd), as shown in Table 1S. The second position is occupied by Química Nova with $5 \%$ of the published papers; $40 \%$ lower than the first position. Although we cannot say that JCEd represents all the research on green chemistry education, it does have the biggest set of documents on this theme, being an important channel to diffuse practices in educational settings at international level. Thinking, accompanying and assisting the development of GC requires its penetration in the training of new generations of chemists, which is a great challenge repeatedly felt by several authors.

The present study has been guided by the attempt to follow the historical international development of GC, investigating what kind of approaches, themes and methodologies towards GC teaching in the perspective of sustainability are present in papers published in the JCEd. It reports a research on papers published by the JCEd until 2019, explicitly affiliated to GC by their authors. It aims to highlight and discuss possible particularities of GC teaching based on proposals and experience reports published in JCEd. Specifically, it is discussed whether GC's closeness to environmental sustainability influences GC teaching, as well as the importance given to systems thinking for the achievement of GC. Investigating GC teaching makes it thus possible to understand how efforts within the field of Chemistry are made towards education for environmental sustainability.

Sustainability/SD, according to the Brundtland Report, ${ }^{5}$ involves three basic components, the so-called $3 P$ : human population (people), economy (profit) and environment (planet). As a methodological option, the analysis here undertaken focused on the environmental dimension of sustainability. It also considered that, fundamentally, GC emerged in order to contribute to solving environmental deterioration, although it did not ignore the importance of the economic factor, which was omitted in the $12 \mathrm{P}$. This omission, incidentally, follows the usual stance of academic chemistry, since the economic aspect of syntheses is rarely mentioned in the respective literature (and also in the literature on chemistry teaching). On the other hand, the social issue is unfortunately the least debated both in GC and in the construction of specific metrics; ${ }^{6,7}$ this observation points to the need for future work to address this issue. In the following, the term "sustainability" refers to environmental sustainability, unless other components are expressly mentioned.

To meet the above objectives, this paper was organized into seven sections, the first being this introduction. Second section, the slow and complex approximation of GC to sustainability, its nature and interrelations, tackles important issues for the definition of the objectives of this research, such as the scarce and tardy approximation of GC to the topic of sustainability, its complexity, and gray areas in defining GC. The third section, GC teaching and its environmental sustainability framework, addresses GC teaching and processes that aim to build a systems thinking-based approach. The fourth section describes the methodological aspects of the research; results are presented and discussed in the fifth and sixth sections, respectively. The last section presents the final remarks.

\section{THE SLOW AND COMPLEX APPROXIMATION OF GC TO SUSTAINABILITY, ITS NATURE AND INTERRELATIONS}

The term CG was coined in the USA, at the EPA, in the early 1990s, when the need was felt to launch a new, intrinsically environmentally benign way to practice chemistry. At the same time, in Europe, the OECD carried out activities to the same end, ${ }^{8}$ but called them Sustainable Chemistry, ${ }^{9}$ which it assumed its relationship with the SD goals laid out in the Brundtland Report.

Even though the $12 \mathrm{P}^{3}$ were proposed in the USA in the same period, they were not (explicitly) linked with sustainability. In the initial phase of GC, only Collins ${ }^{10}$ made that connection (p. 695). Within the IUPAC, in a terminology discussion pitching Green versus Sustainable Chemistry, ${ }^{9}$ an attempt was made to explain an engagement and link of chemistry with sustainability. Only later, in 2002, Anastas, one of GC's precursors, for the first time related GC with sustainability/SD. ${ }^{11}$ In short, GC initially converged with the idea-force contained in the concept of SD, and only progressively was framed within sustainability. ${ }^{12}$

This is still an unresolved issue, as sustainability and SD have been used in parallel and even associated (e.g. Green and Sustainable Chemistry, G\&SC) in a series of congresses named International Conference on Green and Sustainable Chemistry, the most recent of which took place in 2019. This joint phrase has recently featured in journals, in papers published in Current Opinion in Green and Sustainable Chemistry; ${ }^{13,14}$ JCEd has posted a call for papers on G\&SC for a thematic issue. ${ }^{15}$

These examples seem to point to more than a semantic issue and relate to the need to delve into the epistemic identity of GC. Over a quarter of a century after its coming into being, it is necessary to analyze which objectives properly belong to GC, their scope, and scientific and non-scientific (i.e. social, political, etc.) dimensions, ${ }^{16}$ in light of GC's output, methods and concepts; more importantly, it is necessary to assess its commitment to sustainability.

In this context, using the term sustainability related to GC requires greater care. When the field of Chemistry is looking for environmentally cleaner and safer practices, it is more directly focused on one of the dimensions of sustainability: the environment. This implies specific and well-defined goals and responsibilities. On the other hand, one issue that has received little attention is precisely the meaning that is contained in the message and in the very concept of sustainability/SD, i.e. on the real possibility of its reach, ${ }^{17}$ facing the limitations of renewable resources, and those imposed by the Second Law of Thermo-dynamics. ${ }^{17}$ In short, like the aspiration to sustainability, the reach of GC has various limitations.

However, GC's importance to society ${ }^{18}$ and for the economy ${ }^{19}$ has also been increasingly highlighted. Clark et al..$^{20}$ and Marion et al. ${ }^{21}$ highlight the most positive role of GC in a circular economy, as a basis for product innovation by means of design, life cycle improvement and 3R (Renewability, Recycle and Reuse) promotion, but ignore the difficulties of integrating chemistry into circular economy. Sheldon ${ }^{22}$ states that there is a growing interest in green growth and sustainable development, which has focused attention on resource efficiency, and has led to a transition from a linear flow of materials in the economy, the "take-make-use-dispose," to a circular economy, more environmentally friendly, as it saves natural resources. However, the fact that there is no explicit economic component in GC is seen as an important shortcoming for the industry (p. 23). ${ }^{22}$ Nevertheless, the fact that economic calculations are so far inexistent does not prevent and has never prevented them from being incorporated into assessments, therefore, the question seems to be more of a decision on whether or not to do it.

Aspects of the nature and scope of GC have been noted in the literature. ${ }^{12,16,17,23}$ For Eilks and Zuin, ${ }^{13} \mathrm{GC}$ is seen as a scientific, technical and practical tool for achieving Sustainable Chemistry, and this implies that Sustainable Chemistry expands the scope of GC to take into account social, economic and environmental considerations, while GC technically ensures that objectives are achievable (p. eA4). ${ }^{13}$ 
However, this "guarantee" is limited, as it only applies in favorable cases, for any technical tool is always dependent on who uses it, and for what purpose, that is, it has limitations of use. GC is not always feasible-for example, when a toxic reagent is to be substituted in a synthesis, you may not find an adequate alternative. Collins ${ }^{10,23}$ offers a less restrictive view of GC, by suggesting three generic principles for its foundation: (1) protection of life as purpose; (2) interdisciplinarity; and (3) need for long-term implementation, given the complexity of chemistry and the environment. This vision better integrates GC into sustainability (see above), providing cohesion with the ecological and social components of SD, thus expressing a less reductionist understanding than that of GC as a mere tool.

Debates on the name, nature and scope of GC are also not very clear on whether it strongly evokes systems thinking for tackling environmental problems in the context of the complex challenges of environmental sustainability—something Graedel ${ }^{24}$ noted very early on, in an article entitled "Green Chemistry as Systems Science".

After GC's first links with sustainability/SD were made by Anastas, in a report of the ACS Symposium Series held in $2000,{ }^{11}$ he pointed that Chemistry's approach to environmental care moves from circumstantial to intrinsic, when the shift from reactive end-of-line control to the proactive control implicit in GC, intrinsic to chemistry itself is carried out. ${ }^{25}$ However, the $12 \mathrm{P}^{3}$ seem to have been thought one by one, without attention to the systems thinking required by $\mathrm{GC}$, as this was not mentioned at the time of its initial presentation.

This reductionist stance hinders the passage to the intrinsic approach. Later, Anastas ${ }^{26}$ again highlights the importance of the holistic approach in the use of the 12P, despite not making clear how this is to be done. The search for new posture means an important change in the style of thought that, as he himself later stated, ${ }^{19}$ is required to realize the transformation of GC from a way to generate better things (essentially an instrument to generate innovation, with a view to obtaining new performances, functions and efficiencies) (p. 13). ${ }^{19}$

To solve the anthropogenic problems of the environment through GC, whether or not created by Chemistry, it is important to think of it in a systemic way akin to how environment and a sustainabilityoriented civilization work. Therefore, setting GC into action in the perspective of environmental sustainability will require a complex and difficult analysis. Morin ${ }^{27}$ believes that the environment should be thought of as a set of dynamic systems in constant transit with their surroundings, simultaneously producing antagonism and complementarity. In this view, systems represent the basic concept of the complexity, because they cannot be reduced to elementary units, simplifying concepts or general laws. Therefore, systems thinking would be based on a holistic conception opposed to the reductionist Cartesian approach reliant on instrumental rationality. ${ }^{28}$ However, for Morin, ${ }^{27}$ a system is not only made up of parts, it has qualities, properties which are said to be emergent from but not existing in the isolated parts: in other words, the whole is more than the sum of its parts. However, some qualities or properties of the parts are often also inhibited by the whole, so it is also worth less than the sum of the its parts (p. 150-152). ${ }^{27}$

The inclusion of systems thinking in GC would enrich it if it adopted a type of hard-or-soft systemic science approach (Table 1), ${ }^{29-31}$ something that seems to have had little attention (see the discussion section below). The hard approach is used for problems with welldefined data, translatable in numbers, and involves stricter techniques and procedures, in order to provide more objective solutions. In contrast, the soft approach is more intuitive, uses more diffuse and selected tools at the discretion of the analyst, focused on the organization of systems, and seeks understanding/learning rather than problem-solving. The following discussion shows that both approaches are meaningful and applicable to GC. It should be noted, however, that hard and soft approaches are extreme cases, and that intermediate methodologies are possible.

In the field of Ecology, Leff ${ }^{32}$ points that the analysis of the environmental issues requires holistic thinking for the recovery of total reality, which is systemic and complex, and it is, therefore, necessary to find a methodology that is able to re-integrate dispersed knowledge within a unified field, which often requires a soft approach. On the other hand, when considering GC's laboratory and industrial practice, a hard approach is in order (that of industrial ecology, proposed by Anastas and Breen ${ }^{33}$ and Graedel ${ }^{34}$ as a GC tool). Thus, addressing GC with systems thinking seems to mean using a hard approach to chemistry and a soft approach to environment, both of which should be integrated-which helps the analysis on the nature, ideas and purposes that move their output and assessment (green metrics).

This consideration highlights the multiple nature of systems. Bunge $^{35}$ classifies systems into five basic types: natural; social; technical; conceptual, and; semiotic. Each type of system is characterized by its own properties and no type is reducible to another, although they can be composed of items of a different type. Thus, a concrete system can be analyzed in its composition (collection of parts), environment and structure (set of connections or couplings between system components and things in the environment that influence or are influenced by the first).

In short, as all this is still open, GC, viewed as a set of chemical innovations aimed at environmental care and sustainability, is still a field under development. For Constable, ${ }^{36}$ the depth, breadth and variety of innovations in chemistry give hope that chemists and chemical engineers will make significant advances, warning of the many gaps that still exist, for example, the need to incorporate life-cycle systems and thinking into chemistry (p. 60). ${ }^{36}$ This is a consequence of the increasing complexity of chemistry when the environmental effects of its practices, processes and products, which must be present in chemist training. For instance, Orgill et al. ${ }^{37}$ and Matlin et al. ${ }^{38}$ consider the vision of unified chemistry, an one-world chemistry, bringing together its roles in creating scientific

Table 1. Comparison of hard and soft systems. Adapted from Cairns ${ }^{29}$

\begin{tabular}{ccc}
\hline Categories & Hard & Soft \\
\hline Problem & With well-defined solution & With several parallel components \\
Targets & One or more, well defined & Cannot be measured \\
Nature / Background / Approach / Response & To "how" & To what" and "how" \\
Complexity & Deterministic & Non-deterministic, unpredictable and non-specifi- \\
able & Difficult task \\
Existence of validation parameters/metrics & Can be defined & Can be diffuse \\
Boundaries & Well defined &
\end{tabular}


knowledge, transposing that knowledge into useful applications, and responding to global challenges and crises. The central feature of one-world chemistry is interconnectivity with global systems and, therefore, sustainability. The meaning given to this assumption is of interconnection of chemistry that, based on their foundations, concepts and theories, form an intradisciplinary body coming from their various specialties (in reductionist headquarters), but that their processes and products come and form a complex system. Something that requires, therefore, in the first instance an interdisciplinary and holistic treatment and ultimately an approach based on the systemic perspective.

\section{GC TEACHING AND ITS ENVIRONMENTAL SUSTAINABILITY FRAMEWORK}

This progressive framing of GC into sustainability has broadened the field of its socio-environmental justification and added the socioscientific efforts to reach a more sustainable development model. From the point of view of education, this requires reintegrating dispersed knowledge into a unified field, ${ }^{1}$ leading to new ways of thinking and practicing chemistry as a science supporting environmental sustainability; as Anastas et al. (p. 12) ${ }^{19}$ state, "if Sustainability is the goal, Green Chemistry will show the way!"

In fact, in this new stance of Chemistry, the environmental component cannot be neglected. The terms sustainability and environmental sustainability carry distinct but interlinked connotations, because the environment is only one of the three basic dimensions of sustainability. ${ }^{39}$ In this sense, according to Ribeiro and Cavassan, ${ }^{40}$ environment encompasses nature thought or represented, which includes nature as transformed by humankind, i.e. natural and artificial objects and phenomena. Thus, the adoption of the term sustainability unmodified by environmental implies enormous expectations and responsibilities to the scientific output and activities of Chemistry, which in practice are difficult to satisfy, and limit the reach of GC, affecting its approach. This is also important in the teaching and training of chemists, deserving attention in the literature on the teaching of GC and the nature of the links established with environmental sustainability.

A first assessment of GC's penetration into Chemistry Education was presented in $2009 .{ }^{41}$ More recently, other diagnoses have been presented on the subject, ${ }^{13,39,42}$ which converge in pointing to a modest but important growth of interest in research focused on education, especially involving the relation of GC to environmental sustainability. For Vilches and Gil-Pérez, ${ }^{43}$ the emphasis on this relationship is necessary, because education for sustainability remains practically absent from many secondary and higher education curricula worldwide.

One review of literature on the teaching of GC concludes for the need to assess the overall greenness of the practices carried out in teaching laboratories, which reinforce that GC's basic idea is very attractive from the point of view of sustainability, and has been strongly embraced by the Chemistry community, but its practice requires a change of attitude, and the adoption of a paradigm of holistic thinking, which considers the lifecycle of the compound and the process used for its manufacture as the basic unit of reasoning, thus taking its global reach into account. ${ }^{44}$ The concern for the use of holistic thinking for the implementation of GC as a system has been materialized in the construction of holistic greenness metrics that simultaneously assess various dimensions of chemical greenness, as opposed to traditional metrics that are one-dimensional and reductionist (see review in Machado). ${ }^{45}$ These metrics are an example of the use of an intermediate systemic methodology between hard and soft science (see above).
A series of papers, all of which co-authored by Eilks, address GC teaching from the perspective of Education for Sustainable Development (ESD). Burmeister, Rauch and Eilks ${ }^{46}$ discuss the significance of the UN challenges regarding sustainability and what ESD pedagogy will mean for Chemistry Education. The study provides an overview of different models to integrate and reconcile sustainability issues with chemistry education. Drawing upon UNESCO,${ }^{47}$ the authors point that a chemistry teaching-focused EDS must be interdisciplinary and holistic, therefore incorporated in the whole curriculum, and not as a separate topic. Four different basic models are presented, one of which prescribes the adoption of GC principles for laboratory work in scientific education. More recently, Eilks and Zuin ${ }^{13}$ reinforce the argument that GC teaching is within the scope of ESD, considering that G\&SC education should play a prominent role in Chemistry research, teaching and training. Sjöström, Eilks and Zuin ${ }^{48}$ consider the need for eco-reflexive training for the environmentalization of chemistry training curricula, within a sustainability perspective.

Along the same line, other authors relate GC to SD and its importance to outline curricular strategies and the production of teaching resources. Haack and Hutchinson ${ }^{49}$ point that in curricular reforms of undergraduate programs and teaching resources, GC insertion will be critical to encourage future innovations in industrial research and applications that meet society's growing demands for sustainable products and processes. In this sense, they highlight that over 200 papers on GC were published in JCEd since 1994, which form a basis to produce new resources and curricular reforms. Since 2001, JCEd has maintained a section for the dissemination of interdisciplinary courses, laboratory experiments, demonstrations and student research. The authors suggest that all this material forms a basis for the construction of a roadmap to facilitate GC teaching.

On the other hand, Płotka-Wasylka et al. ${ }^{50}$ mention several important dimensions in GC education, which should be taught at all levels to press societal shift towards sustainability. They even suggest teaching in Humanities, for example, by integrating GC with non-scientific modules in fields such as Law, Ethics or Business, or even the "greening" of courses in these fields.

Marques and Machado ${ }^{43}$ point that GC education would be strengthened if rooted in the construction of an ecological culture from the school, in line with environmental education, which requires a repositioning of all scientific fields, aiming at an interdisciplinary approach and a critical vision of current science-non-reductionist, contextualized and integrated in the social, historical and political dimensions.

In conclusion, some trends are noticeable in the literature, albeit dispersedly presented, of proposals on the GC teaching committed to sustainability/SD, with variations regarding its penetration in chemistry curricula and the pedagogical perspectives. In addition, the connection of GC with SD is sometimes treated as synonymous with sustainability, without much attention being paid to the environmental component. In order to obtain a more detailed view of the abovesketched panorama, we decided to make an analysis of the literature on GC published in JCEd, as it is the main vehicle for chemistry teaching research output.

\section{METHODOLOGY}

This study conducted a survey and selection of GC educationrelated papers published in the JCEd; it was conducted in the journal's website, spanning until the end of 2019. Initially, the search term "Green Chemistry" was sought in paper titles, abstracts and keywords. After editorials, news, communications etc. were removed from the sample, there were found 286 texts identified as research papers. 
The classification of contents was made in these 286 papers by means of previously established categories (based on Marques and Machado $)^{42}$ related to: source-problem, paper focus, subjects/area of knowledge, teaching and training target groups, GC contents, type of approach and purpose(s) of the proposal. These categories express a set of aspects that identify what, where/what for, how and for whom each paper is intended, and how they establish links between GC and sustainability/SD, a systemic vision, in addition to identifying particularities of GC teaching. All the papers were fully read, excerpts were highlighted, categories were created based on reading, followed by grouping of texts and filling out the analytical table, available in Supplementary Information (Table $2 S$ ).

\section{RESULTS}

The discussion was held evaluating the results obtained in the composition of contents of each category. In most cases, a list was presented prior to the analysis with a set of examples of the features of each category, followed a discussion thereof, which compares the information found with the category's list of features (with the caveat that the features from a previously defined list were not always found in the results, and vice-versa), which are discussed below. The purpose of this procedure is to provide a less rigid view of the features of the a priori categories, and also the possible emergence of others. This methodology, although soft, has made it possible to highlight the most significant results to bring light to our objectives.

The proportion of GC teaching papers that explicitly mentions sustainability has grown from $13 \%$ in the period 2003-2006 to $68 \%$ in 2019 (Figure 1).

The "Paper Focus" category (see Table 2) allows an overview of the nature of the paper: whether literature review (state-of-theart), experience report, didactic proposal or theoretical essay. Three reviews were found; most papers (188) provided experience reportstherefore, a work that has already been carried out, with a type of approach focused on the development of practical activities related to experimentation and chemical demonstration. This type of activity is very significant, as it shows an action involving teaching and an effort by researchers to report-and, in some cases, assess-their educational experience. A significant number of papers also focused on pedagogical proposals (88), whether derived from experiences or not; in the first case, they function as teaching suggestions, using as examples something carried out by other subjects. Finally, only one paper was characterized as theoretical, anchored in a literature analysis.

Table 2. Distribution of Papers According to the Focus and Use of the Term Sustainability

\begin{tabular}{lc}
\hline Paper Focus & Frequency \\
\hline Experience report & 188 \\
Teaching proposal & 88 \\
Theoretical essay & 7 \\
Review & 3 \\
\hline Total & $\mathbf{2 8 6}$ \\
\hline
\end{tabular}

The "Paper Source-problem" category includes features such as: (i) identification of problems in chemistry education that would require a $\mathrm{GC}$ or environmental perspective in its approach; (ii) environmental issues that, in educational contexts, would be best addressed by GC; (iii) the need of GC in teaching because it is linked to sustainability; (iv) resolution of socio-environmental problems, everyday issues, socio-scientific issues; (v) specific problems of GC; and (vi) the use of GC principles and procedures to make chemistry "cleaner".

The results show a concentration of papers in the following source-problems: denounce the lack of educational material related to the GC/sustainability (98) (feature I); use of chemical processes with solvent reduction (39) (features iv and vi); a characterization of the existence of hazards (32) (iv and vi); the contribution of chemistry to the environmental crisis (25) (ii); and the need for chemistry greenness assessment (18) (vi). Increasing GC knowledge and developing materials for industry relate to characteristic (v), with 10 papers (see Table 3). Comparison with the list of problems of the category under consideration shows that characteristic (iii) may be present in approach of the environmental crisis (25), energy crisis (12) and impact of fuels (2). Although the highest number or papers focused on teaching resources, complaints were often found in the literature that there is little or no access to GC teaching-related resources. ${ }^{49} \mathrm{It}$ also shows that priority focuses coincide with the literature of GC in general, such as the reduction of solvents, hazards in chemistry and the negative role of chemistry in the emergence of environmental issues.

The "Type of Approach" category (see Table 4) sought to include the proposals, assessments and/or reports involving GC teaching

\section{No mentions Mentioning sustainability}

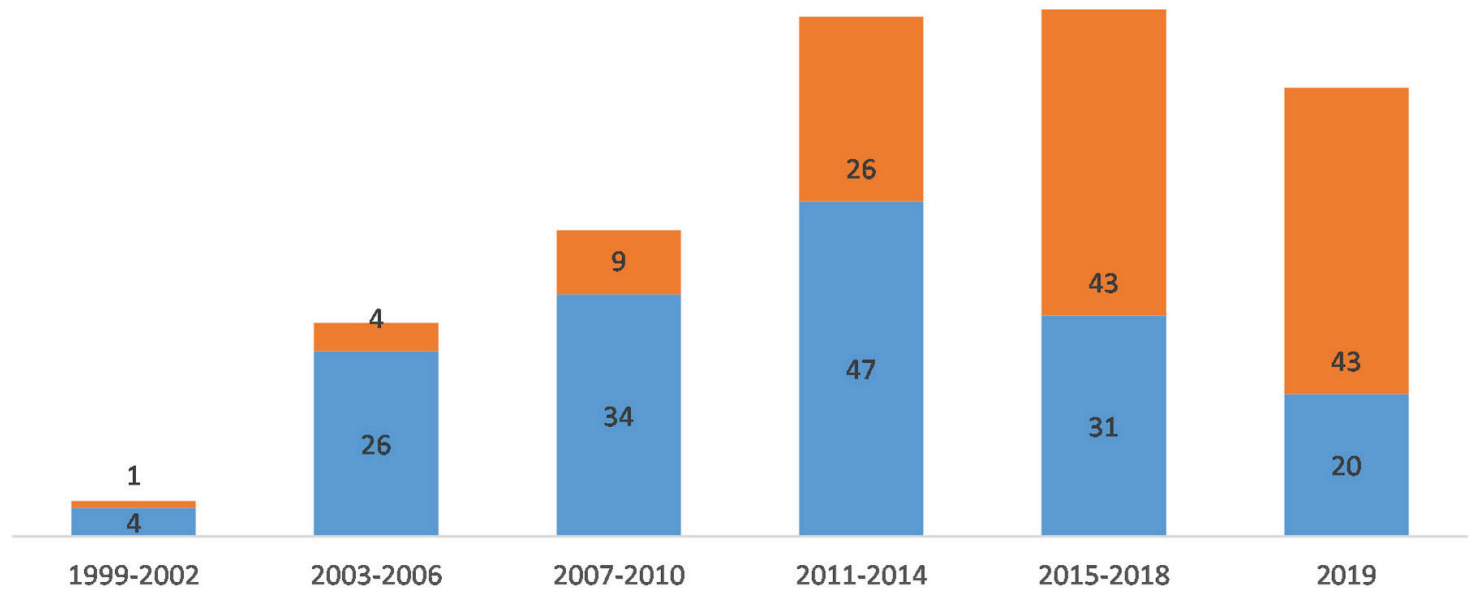

Figure 1. Distribution of GC education papers published in the JCEd by year, and by use of the term sustainability 
Table 3. Source-problem on GC Teaching Research

\begin{tabular}{lc}
\hline Source-Problem & Frequency \\
\hline Absence of Green Teaching Material & 98 \\
Solvent reduction & 39 \\
Dangerous process & 32 \\
Environmental Crisis & 25 \\
Full education of chemists & 21 \\
Greeness assessment & 18 \\
Systems complexity & 13 \\
Energy crisis & 12 \\
Increasing GC knowledge & 7 \\
Waste disposal & 4 \\
Introduction to GC & 3 \\
Curriculum & 3 \\
Development of new material to industries & 3 \\
Reagent replacement & 3 \\
Evaluation and recycling of catalysts & 2 \\
Environmental impact of alternative fuels & 2 \\
Not defined & $\mathbf{2 8 6}$ \\
\hline Total &
\end{tabular}

strategies formulated and discussed in each paper. In this sense, we consider the models of curricular insertion of educational activities aimed at sustainability or GC. ${ }^{46,51}$ The following types of approach are predominant: (i) punctual insertions (GC teaching of a given GC content or GC taught at a specific moment); (ii) offer of a specific GC subject; (iii) the development or suggestion of an experimental activity; (iv) cross-sectional GC insertion in the chemistry curriculum; (v) GC teaching associated with socio-scientific aspects (Table 4).

The results point to the predominance of punctual GC content insertions in courses, both theoretical and practical, with a predominance of laboratory activities (199), including demonstration and illustration (features $\mathrm{i}$ and iii). Approximately $10 \%$ of the
Table 4. Type of Approach

\begin{tabular}{lc}
\hline Type of Approach & Frequency \\
\hline Laboratory insertion & 199 \\
Transdisciplinary & 17 \\
Curriculum & 14 \\
Practical course & 14 \\
Theoretical and practical course & 12 \\
Theoretical and practical insertion & 12 \\
Demo/Illustration & 7 \\
Interdisciplinary & 4 \\
Non-Formal/Minicourse & 2 \\
Non-Formal/Practical course & 2 \\
Minicourse & 1 \\
Non-formal & 1 \\
Online Course & 1 \\
\hline Total & $\mathbf{2 8 6}$ \\
\hline
\end{tabular}

papers show feature (ii, offer of a specific GC subject): practical courses (14), theoretical and practical courses (12), non-formal/ practical courses (2), non-formal/minicourse (2) and minicourse (1). Finally, $8 \%$ of the papers address GC in the curriculum in a trans or interdisciplinary way (17 and 4 , respectively) (feature iv).

Information on the focus of the papers and the type of approach was cross-checked in order to quantify correlations between the GC teaching strategies and the nature of research (see Table 5). Most of the teaching proposals (66) and experience reports (133) dealt with laboratory experiments, with a total of 199 papers. Experience reports on trans or interdisciplinary practices compose the second largest set (13). Most proposals for laboratory insertion involved organic chemistry, an aspect that has also been repeatedly highlighted in the literature. ${ }^{44,52,53}$

With regard to the subjects and/or fields of Chemistry the papers under analysis address (see Table 6), the vast majority refers to classical areas, such as Organic Chemistry (151) and General

Table 5. Relationship between Type of Approach and Paper Focus

\begin{tabular}{|c|c|c|c|c|c|}
\hline \multirow[b]{2}{*}{ Type of Approach } & \multicolumn{5}{|c|}{ Focus Papers } \\
\hline & $\begin{array}{c}\text { Theoretical } \\
\text { Essay }\end{array}$ & $\begin{array}{l}\text { Didactic } \\
\text { proposal }\end{array}$ & $\begin{array}{c}\text { Experience } \\
\text { report }\end{array}$ & Review & Total \\
\hline Laboratory insertion & & 66 & 133 & & 199 \\
\hline Transdisciplinary & & 7 & 10 & & 17 \\
\hline Curriculum & 6 & 1 & 4 & 3 & 14 \\
\hline Practical course & & 1 & 13 & & 14 \\
\hline Theoretical and practical course & & 3 & 9 & & 12 \\
\hline Theoretical and practical insertion & & 7 & 5 & & 12 \\
\hline Demo/Illustration & 1 & 2 & 4 & & 7 \\
\hline Interdisciplinary & & 1 & 3 & & 4 \\
\hline Non-Formal/Practical Course & & & 2 & & 2 \\
\hline Non-Formal/Minicourse & & & 2 & & 2 \\
\hline Online Course & & & 1 & & 1 \\
\hline Minicourse & & & 1 & & 1 \\
\hline Non-formal & & & 1 & & 1 \\
\hline Total & 7 & 88 & 188 & 3 & 286 \\
\hline
\end{tabular}


Chemistry (45), with the remainder dispersed among other areas (or subject denominations); two papers are specifically devoted to GC as a subject (2). Fuzziness among areas and subjects does not follow a single pattern: for example, the fields of organic and inorganic catalysis, depending on the curricular organization, may or may not be combined to those of organic and inorganic chemistry, respectively. This may explain why the number of subjects associated with catalysis is low, despite its importance within GC.

Table 6. Subject/Field of Chemistry

\begin{tabular}{lc}
\hline Branch of Knowledge & Frequency \\
\hline Organic Chem & 151 \\
General Chem & 45 \\
Chemistry & 23 \\
Analytical Chem & 16 \\
Inorganic Chem & 14 \\
Interdisciplinary & 6 \\
Physical Chemistry & 6 \\
Teaching & 5 \\
Industrial Chem & 4 \\
Environmental Chem & 2 \\
Green Chemistry & 2 \\
Inorganic catalysis & 2 \\
Organic catalysis & 2 \\
Polymers & 2 \\
Thermodynamics & 2 \\
Biochemistry & 1 \\
Electrochemistry & 1 \\
Ethics & 1 \\
Management and Marketing & 1 \\
\hline Total & 286 \\
\hline
\end{tabular}

The analysis also provided an overview of the category "GC Content" in education (see Table 7). An expressive number of papers refers directly or indirectly to the $12 \mathrm{P}$ (69); others, to the development of safe, clean or benign synthesis, process and products (62). The substitution of solvents (28) and proposals or reports on how to work GC examples in chemistry teaching (27) are also expressive, and so are papers on education about GC in general (16), about simple processes suitable for educational environments (16), and metrics (12), although to a lesser extent. The relatively high number of papers referring to the $12 \mathrm{P}$ is not surprising, given their importance in the dissemination of GC. The number of papers on catalysts and metrics seems low in relation to their importance in the implementation of GC.

The "Teaching and Training Target-groups" (see Table 8) gathers information on the main recipients of the education proposals in each paper, especially the level of Education: Elementary, Secondary or Higher Education, non-formal education, and/or interfaces among them. The analysis shows a very wide diversity in target audiences, and a variety of sometimes integrated levels of education.

Almost $90 \%$ of papers address undergraduate courses, followed by $9 \%$ on inserting GC in High School or education in general. Teachers education is explicitly referred in two papers. Several proposals broadened their range (suggesting that they involve interface among levels), and several did not make clear their targets (one such paper targeted the general public). This imprecision as to
Table 7. GC Content of Teaching

\begin{tabular}{lc}
\hline GC Content & Frequency \\
\hline GC Principles & 69 \\
Solvent Replacement & 28 \\
GC examples & 27 \\
Benign or safe synthesis & 25 \\
Less dangerous processes & 23 \\
Simple processes & 16 \\
Clean processes & 13 \\
GC Education & 13 \\
Metrics & 12 \\
Renewability & 11 \\
Waste management & 10 \\
Catalysis & 8 \\
Not defined & 7 \\
Life Cycle & 6 \\
Alternative source of energy & 12 \\
New reaction procedures & 4 \\
Use of microwaves & 1 \\
Catalyst recycling and reuse & 4 \\
Safe product design & 1 \\
Sustainable consumption & 1 \\
System Diagrams & 1 \\
Teaching/Learning & 1 \\
Use of less reagent & 1 \\
Total & 1 \\
\hline & 13 \\
\hline
\end{tabular}

Table 8. Teaching and Training Target-Groups

\begin{tabular}{lc}
\hline Main Teaching Subjects & Frequency \\
\hline Undergraduate course & 247 \\
High School & 26 \\
General Education & 20 \\
Not defined & 6 \\
Graduate course & 5 \\
Basic education & 4 \\
Teachers Education & 2 \\
Elementary School & 1 \\
Technical high school & 1 \\
Higher education & 1 \\
\hline Total & $\mathbf{3 1 3}$
\end{tabular}

Note: total number is bigger than 286 , as each paper may address multiple target-groups.

the level of education and target groups may indicate problems in reach and quality of the suggested training.

The "Purpose of Proposals" category (see Table 9), present each of the 286 articles, involves considering their scope or reach, by identifying characteristics such as: (i) teaching GC concepts and comparing them with those of Classical Chemistry; (ii) solving socioenvironmental problems from the perspective of GC; (iii) teaching experimental procedures; (iv) teaching GC principles and concepts; 
Table 9. Purpose of Proposals

\begin{tabular}{lc}
\hline Purpose of proposals & Frequency \\
\hline Comparing Classical Chemistry with Green Chemistry & 23 \\
Comparing Classical Chemistry with Green Chemistry - Metrics & 6 \\
Curriculum comparison & 2 \\
Development of green or sustainable products & 1 \\
Development of green skills & 61 \\
Development of systems thinking & 13 \\
Improvement and creation of teaching material & 104 \\
Others (informative) & 1 \\
Promoting Chemistry Education & 39 \\
Promoting Green Chemistry Education & 12 \\
Socio-scientific approach & 6 \\
Tackling environmental issues & 18 \\
\hline Total & $\mathbf{2 8 6}$ \\
\hline
\end{tabular}

(v) solving problems related to the chemist training from the perspective of GC; and (vi) solving problems of Chemistry in general. The results again show the strong tendency to consider the need for improvements in chemistry teaching resources for the teaching of GC (104) (ii, iii, iv and v). They also highlight the need to develop "green" skills in students (61) (ii, iv and v). GC was referred as an important knowledge for chemistry education in general in 39 papers. Some papers compared GC and Classical Chemistry (29); among these, some claimed for metrics to differentiate them (i) (6 papers). This is important if we take into account the discussion in the literature about what is or is not GC. ${ }^{16}$

When Purposes and Source-problems are compared (see Table 10), one of the biggest problems seem to be the lack of teaching resources (98 papers), which is addressed so as to improve and chemistry teaching materials (49)—without a clear distinction between creating new resources or simply improving existing ones-and the development of "green" skills (27). Improving existing teaching resources was also a solution found to propose reductions in solvents (25) and hazardous processes (20). Chemist training is a much more recurring objective than promoting GCspecific training, which demonstrates or would justify the oftensuperficial incorporation of GC into these papers, an addendum or extra quality of the reported proposals rather than a specific objective. Proposals thus made might imply that, for these authors, GC would not be a specialty or separate discipline within Chemistry: it is the full chemist training that needs to incorporate the knowledge and practices under GC.

The survey identified 39 articles related to systemic thinking and the teaching of Green Chemistry, which includes the 30 articles published in 2019 in the special issue on systemic thinking in the teaching of green and sustainable chemistry. ${ }^{54}$ More than half of those papers are experience reports. The six theoretical essays found in this whole research are about systems thinking.

Table 10. Relationship between Purposes and Source-problems

\begin{tabular}{|c|c|c|c|c|c|c|c|c|c|c|c|c|c|}
\hline Source-problem & $\begin{array}{l}\mathbb{x} \\
\text { n }\end{array}$ & $\begin{array}{l}\dot{\Xi} \\
\text { Uे } \\
\text { 官 }\end{array}$ & 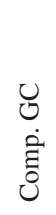 & 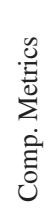 & 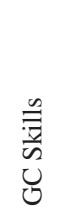 & 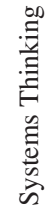 & 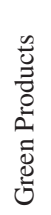 & $\begin{array}{l}\dot{\infty} \\
\dot{0} \\
\dot{\overrightarrow{0}} \\
\dot{=1}\end{array}$ & 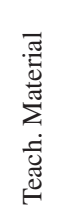 & $\frac{\tilde{\omega}}{\tilde{0}}$ & 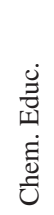 & 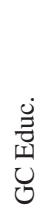 & 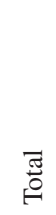 \\
\hline Absence of green teaching material & & 1 & 5 & & 27 & & & 6 & 49 & & 9 & 1 & 98 \\
\hline Solvent Reduction & & & 5 & 1 & 7 & & & & 25 & & 1 & & 39 \\
\hline Hazardous process & & & 3 & & 3 & 1 & & 1 & 20 & & 3 & 1 & 32 \\
\hline Environmental Crisis & 4 & & & & 6 & & & 8 & 3 & & 4 & & 25 \\
\hline Full education in Chemistry & 2 & & & & & 1 & & & & 1 & 14 & 3 & 21 \\
\hline Greeness evaluation & & & 5 & 5 & 7 & & & & 1 & & & & 18 \\
\hline Systems Complexity & & & & & & 10 & & & 1 & & 1 & 1 & 13 \\
\hline Energy crisis & & 1 & 1 & & 5 & & & 3 & & & 2 & & 12 \\
\hline Improving GC Knowledge & & & 1 & & & & & & 1 & & 1 & 4 & 7 \\
\hline Waste disposal & & & & & 1 & & & & 2 & & & 1 & 4 \\
\hline Introduction to GC & & & 1 & & 1 & & & & & & & 1 & 3 \\
\hline Curriculum & & & & & 1 & 1 & & & & & 1 & & 3 \\
\hline Developing new materials for industry & & & & & 1 & & 1 & & 1 & & & & 3 \\
\hline Reagent replacement & & & & & 1 & & & & & & 2 & & 3 \\
\hline Catalysts Evaluation and recycling & & & 1 & & & & & & 1 & & & & 2 \\
\hline Environmental impact of alternative fuels & & & 1 & & 1 & & & & & & & & 2 \\
\hline Not defined & & & & & & & & & & & 1 & & 1 \\
\hline Total & 6 & 2 & 23 & 6 & 61 & 13 & 1 & 18 & 104 & 1 & 39 & 12 & 286 \\
\hline
\end{tabular}

SSA: Socio-scientific approach; Comp. Curr.: Curriculum comparison; Comp GC: Compare Classical Chemistry with Green Chemistry; Comp Met: Compare Classical Chemistry with Green Chemistry - Metrics; GC Skill: Develop green skills; Systems Thinking: Developing systemic thinking; Green Products: Development of green or sustainable products; Envir. Iss.: Tackling environmental problems; Teach. Material: Improvement and creation of teaching material; Others (informative); Chem Educ.: Promoting Chemistry Education; GC Educ.: Promoting Green Chemistry Education. 
Regarding the source-problem for inserting systems thinking in (green) chemistry education, one third of papers indicates the complexity of issues addressed by chemistry, such as the environmental crisis, pollution and achieving sustainability. Also, one third of papers discuss a curricular type of approach for systems thinking, considering Chemistry in general as the related branch of knowledge, and developing systems thinking as a purpose for the educational proposals. Industrial chemistry and interdisciplinary approaches ( 3 papers each) are also appointed as subject matters. Promoting education on general topics of GC (5 papers), addressing socio-scientific issues (3) and promoting full education of chemists (3) are also frequent purposes. Educating on GC in general is the most frequent (10 papers) educational content related to systems thinking, followed by life-cycle issues (4). Most of the papers discussed systems thinking in undergraduate courses (13), followed by 5 papers directed to education in general. Full data is available as Supplementary Information.

Several authors argue that the adoption of Systems Thinking should be a complement to the reductionist approaches to science. ${ }^{37}$ Most articles reported teaching experiences (20) (e.g. Aubrechet et al. $)^{55}$ or didactic proposals (2) using Systems Thinking and some only in the form of theoretical essays (5) (e.g. Aubrecht et al.). ${ }^{55}$ Review articles (3) (e.g. York et al. $)^{56}$ on the use of conceptual structures of Systems Thinking in other areas of teaching or training fields (life sciences, earth sciences and engineering) are presented as suggestions of transposition to the teaching of (green) chemistry, but pointing out that this needs to be researched and that experiences need to also consider how to evaluate the benefits and results in the use and learning of students. Other articles (4) highlight gaps in learning in disciplines and point out the potential of systemic approaches when associated with some learning theories (such as Pazicni and Flynn). ${ }^{57}$ The majority of the articles present broader curricular formulations and not only specific disciplines, formulated for the various levels of education and others (8) deal with specific activities to the teaching laboratory. One article ${ }^{58}$ associates the Science-TechnologySociety-Environment approach (CTSA approach as in portuguese) as a big step toward a more global systems thinking approach. Some articles (9) relate green and sustainable chemistry to circular economy, emphasizing the need for Systems Thinking as the best approach to better understand their interrelationships. Mammino ${ }^{59}$ emphasizes the importance of language and its mastery in scientific education for the understanding of complex systems. Miller ${ }^{60}$ applied Systems Thinking in educational games to facilitate a learning approach involving recycling processes and green chemistry, in accordance with the United Nations Sustainable Development Goals.

Perosa et $a l .{ }^{61}$ brings the concept of Emergy, first developed by Odum, ${ }^{62}$ to quantify the energy involved in transformations of a system to produce a product or services, which generate energy memories by emerging flows, which aid in the survival or transformation of these systems. It exemplifies the concept in the analysis of glass craft production venture in Venice, Italy. The article makes use of systems diagrams and employs a simulator that calculates the capacity depending on the state variables to indicate the evolution of the system and the energy undertaken. The authors argue, in general, that the Systems Thinking paradigm, when combined with the student-directed activities, will create in them the interest in learning how things work and can change. To support this hypothesis, several research and experiences of CG are used, dynamically linking chemistry to the theme of sustainability.

Most of the texts are concerned with presenting Systems Thinking conceptually, describing its origins and characteristics, to emphasize interconnections with GC and to explain the relationship with sustainability. Most of the papers in the special issue has
Mahaffy et $a .^{63}$ and Matlin et al. ${ }^{38}$ as supports to the concept of systemic thinking. Orgill et al. ${ }^{37}$ make a summary on systems thinking concept based on Richmond ${ }^{64}$ and the aforementioned vision of oneworld chemistry. However, there were also those who, like Murphy, ${ }^{65}$ used the IUPAC as a reference for Systems Thinking in Chemical Education ${ }^{66}$ and the International Organization for Chemistry Sciences in Development. ${ }^{67}$

The texts reflect this effort with arguments and examples about some ways of systemically connecting the components that interact and are involved in chemical processes, their internal and external boundaries, as well as changes in forces related to their underlying dynamics. ${ }^{64}$

\section{DISCUSSION}

The analysis undertaken has shown a wealth of GC teaching approaches and experience reports (Table 3), most of which (199) related laboratory activities for teaching, mostly organic chemistry (151) (Table 5) and targeting undergraduate courses (247) (Table 7), by inserting GC Principles (69) or providing examples thereof into classical chemistry syllabi (27) (Table 6). Crossing categories and their characteristics (Tables 4 and 9) shows more clearly the concentration of papers related to chemical experiments, besides proposals and concerns about GC teaching resources. It is also important to note that, although the papers mention that they employ new chemical practices which they call $G C$, not all papers detail, deepen and problematize the way in which they are developed into teaching situations.

It was difficult to find evidence of the particularities or specific characteristics of GC teaching, that is, even though JCEd specializes in chemistry teaching, the papers under analysis, in general, did not go into details on their pedagogical point of view and teaching situations, which could have qualified the work set out in the paper. Several authors only highlighted, with some examples, successful cases or GC's potential for application, and how necessary it is in teaching, often justified to materialize and increase the chemistry's commitment to environment and sustainability. Not infrequently, there seems to have been a certain degree of reproduction of traditional chemistry teaching models, as no details were offered on how to increase chemical greenness.

Nonetheless, the variety of contents presented and subsumed under GC by the authors signal the penetration of GC research into chemistry teaching. Although boundaries among research areas in GC are still somewhat fuzzy, ${ }^{12,20}$ the proposition of GC contents (Table 8) to be addressed by/in the traditional chemistry curricula and subjects indicates a path of improvement for chemist training towards environmental preservation.

Additionally, most papers did not provide information and evidence to further understand the relationship between GC, (environmental) sustainability and SD, and even employ different terms to affirm green practices or their effectiveness. Most of the papers addresses GC and its practices as a means to achieve sustainability. For instance, Mahaffy et al. ${ }^{68}$ states that systems thinking is useful to educate on the molecular basis of sustainability. On the other hand, Eaton et al. ${ }^{69}$ highlights that systems thinking was responsible to direct Australian curriculum towards sustainability. Lasker et al. $^{70}$ believes sustainability and other environmental sciences provide knowledge needed to a greener practice. Nevertheless, the relationship between GC and sustainability issues are not explicit or self-evident and need to be clearly addressed, as pointed out by Josephson et al. ${ }^{71}$

Teaching experiences and proposals are not always presented alongside detailed suggestions for their implementation. This may 
be indicating that there are still difficulties in the implementation of GC teaching; to put it briefly: we may know what to do, but not yet how to do it.

In addition, we also note that few papers mentioned the need for GC to have a systemic or holistic thinking base in addressing environmental problems and chemist training..$^{53,72-79}$ The analysis of this set of articles showed that different forms and understandings about the application of this theoretical support, called systemic thinking, aiming at a holistic approach in the teaching of green chemistry. In some cases, it was even incipient, staying more in advertising and prescriptive aspects. This is understandable, considering its complexity, the involvement of different areas of knowledge and the reductionist matrix of our scientific formation. According to Mahaffy et al. ${ }^{80}$ (p. 364) the systemic perspective that relates chemical activities to sustainability challenges is not yet fully or systematically incorporated into routine chemical practice.

In the teaching of green and sustainable chemistry, there are several fields and areas of knowledge involved, such as education and teaching, natural sciences, engineering and environmental sciences, forming a complex of specific conceptual and methodological systems. Some articles, in their foundations and propositions, recognize and give treatment to these scopes and complexity. In this sense, Bunge's five basic types of systems can help classifying and organizing this complexity. ${ }^{35}$

The indicators obtained in the analysis of the JCEd papers are still insufficient to identify methodological trends more congenial to GC teaching, which means our main objective was not fully attainable. However, the literature on chemistry teaching from an environmental perspective, whether or not inserted in GC and connected with sustainability/SD, already provides some paths in various dimensions to be followed for a GC education. In the curricular aspect, the CTSA approach $^{47}$ and the environmentalization ${ }^{81}$ of teaching programs and chemistry curricula must be considered as a whole. Regarding greater technical eco-efficiency, the $12 \mathrm{P}$ remain an important reference, even if there is little research on an integrated approach to them; additionally, they may still be insufficient for the long-awaited reach of the chemical greenness. GC's commitment to sustainability also needs to incorporate social and scientific aspects expressed in UN's 17 SDGs. ${ }^{82,83}$ As for the epistemological aspects, a more consistent analysis of how to build a systemic vision of GC aspirations-from the molecule design to product processes and lifecycles in the environment-, and always with the support of consistent metrics, may help its consolidation as a change in the rationality of chemicals and their effective commitments to sustainability.

These elements are important for an eco-reflexive ${ }^{84}$ and humanistic ${ }^{76}$ chemistry, the teaching of which is yet to be developed and assessed. But to provide a professional chemist training in these perspectives requires a basis that intertwines various dimensions of knowledge. In this sense, the humanist perspective is characterized and structured by Sjöström and Talanquer ${ }^{76}$ as a gradation of relationships between the chemical and critical-reflexive approaches, summarized in the following: pure chemistry (exclusively conceptual); applied chemistry (daily life uses); socio-cultural chemistry (providing epistemological, social and historical descriptions of knowledge in chemistry for scientific decision-making); a critical-reflexive chemistry (problematizing chemistry's knowledge and practices, stressing its values and directed to transforming action).

We view a critical-reflexive chemistry training ${ }^{49}$ as fundamental to the constitution of an environmental rationality ${ }^{32}$ within the Chemistry community. However, there are many obstacles to this: the modernist discourse on Chemistry ${ }^{85}$ technological optimism on control over nature; scientific objectivism of knowledge, which would be independent of historical and social factors; the reductionism of entities' properties, and the rationalism of strictly "objective" decision-making, which disregards ethics and politics. From the point of view of chemical education, such obstacles show, for example, in content - whose focus is fixed on theoretical concepts rather than historical practice-, and in strengthening theoretical-contemplative "modes of reasoning" and instruments of domination. Therefore, it involves different rationalities, in processes constituted historically and socially, which are difficult to change by the sheer socialization of successful works and ideas, requiring theoretical and practical training.

Finally, two related points are worth mentioning. First, about a quarter of the papers under analysis adopted distinct denominations for $\mathrm{GC}$, thus reinstating the issue around the lack of terminological uniformity. ${ }^{13}$ In this context, we agree with ${ }^{86}$ that it is more important for chemists to apply their knowledge, skills, creativity and intuition consciously and continuously to help anticipate and minimize the human impact on the environment in which we live, regardless of "whether such chemistry should be called 'green', 'clean', 'cleaner' or 'sustainable' or simply continue to be called chemistry" (p. 8) ${ }^{86}$ Caution seems necessary when naming GC, lest we are led by simplifications, and carelessly disregard the implications and difficulties related to the delimitation of its boundaries, attributing to chemistry as a whole demands and responsibilities linked to the various dimensions of sustainability. It should also be noted that we need to consider the entropic limits, ${ }^{87,88,17}$ the idea of prevention, and the need to have a better knowledge about the toxicity of substances in the environment, the conditions attached to the principles of prevention and precaution, ${ }^{89}$ without ignoring the barriers to the effectiveness of the technique.

Second, difficulties were found in the JCEd paper search system itself. For the issues published in the initial phase of the journal (online), there were no keywords or even abstracts, which eventually causes some entropy in the database. The adoption of criteria identifying content, provided by modern bibliometry, could help in state-of-the-art researches, especially in innovative fields of knowledge, of which GC is a representative.

\section{CONCLUSIONS}

In seeking to identify descriptive elements in the papers on GC in JCEd, the intention was to obtain information that would reveal what GC should be and what GC really is. It may be instrumental in helping teachers and researchers find ideas and teaching experiences, for example, to differentiate between the theoretical rationality (devoted to the explanation of what it is like in the world) and practical rationality (which intervenes in the world, and how it should be), ${ }^{28}$ which are supposed to be crucial to GC, as they make it possible to assess the role of this field of science for sustainability, and ecosocial and environmental justice. ${ }^{48,84}$ Keeping this in mind would help identifying qualitatively possible trends in the teaching of GC/ Sustainability, whether or not reinforcing a holistic or instrumental/ reductionist perspective. However, this study showed that it was difficult to find methodological trends specific to GC teaching, despite the existence of literature on GC, chemistry teaching and sustainability/SD, which may assist in GC education.

It is worth mentioning that the scope of the JCEd is directed to provide resources to chemistry educators and their institutions. This played a major role in the number of experience reports and teaching activities found. Although papers published in other journals may address the green chemistry education in a more complex and deep manner, it is remarkable that $46 \%$ of the scientific outcome on the theme is published in the JCEd. It may indicate a trend in green chemistry education towards application of incremental green 
practices, rather than a substantive transformation of the activities. More research in other sources are needed to fully clarify this issue.

Accordingly, the analysis of articles related to systemic thinking highlights the complexity and challenge of its application in approaches to teaching green and sustainable chemistry. Perhaps this is a reflection of its little use in the field of chemistry itself, even when inserted in the perspective of sustainability. Therefore, Systems Thinking is a new and promising challenge for G\&SC, especially for its teaching. It is something innovative from a pedagogical and methodological point of view, deserving greater analytical and investigative depth.

The penetration of GC into the curriculum and the initial training of chemists is undeniable. But the reformulation of chemistry so as to embrace and address the multiple dimensions of sustainability, and the new practices and conceptual issues proposed by GC cannot ignore the need for the training of a specific type of teacher, whose actions need to be infused with a broad social-political intention (facing environmental issues), as teachers are bearers of the projects whose practices denote social ends. ${ }^{90}$ In this sense, in university chemist training (as chemistry teachers or chemists), when conducted by a curriculum that includes $\mathrm{GC} /$ sustainability and educational practices, ${ }^{91}$ needs to be critical-reflexive in order to contribute significantly to an environment culture for future teachers who will act in basic education, thus forming a virtuous circle.

\section{SUPPLEMENTARY MATERIAL}

Number of documents published by journals according to Web of Science database (Table 1S). Analytical data of papers and complete references (Table 2S) are freely available in pdf format at http:// quimicanova.sbq.org.br.

\section{ACKNOWLEDGEMENTS}

We would like to thank CNPq for Projeto Universal-405034-2016-4 and the grant of scholarhips; Capes for funding doctoral, sandwich doctoral (Capes/PDSE/Process n. 88881.189651/2018-0) and postdoctoral scholarships abroad; FAPESC/SC-Brazil for the Doctoral Fellowship. We would also like to thank the support of Fabiana Roberta Gonçalves e Silva Hussein, Kelly Bernardo De Paulo, Marines V. Ferreira, Nicolle Ruppenthal.

\section{REFERENCES AND NOTES}

1. Leff, E.; Epistemologia Ambiental, Cortez: São Paulo, 2001.

2. Ortega y Gasset, J.; Meditação da técnica, Livro Ibero-Americano: Rio de Janeiro, 1963.

3. Anastas, P. T.; Warner, J. C.; Green Chemistry: Theory and Practice, rev. ed., Oxford University Press: USA, 1998.

4. Anastas, P.; Green Chemistry Letters and Reviews 2007, 1, 3.

5. WCED; Our Common Future, $1^{\text {st }}$ ed. Oxford University Press: New York, 1987.

6. Iofrida, N.; Strano, A.; Gulisano, G.; De Luca, A. I.; Int. J. Life Cycle Assess. 2018, 23, 201

7. Sierra, L. A.; Yepes, V.; Pellicer, E.; J. Cleaner Prod. 2018, 187, 496.

8. OECD; Proceedings of the OECD workshop on sustainable chemistry - Part1, OECD: Venice, 1998

9. Tundo, P.; Anastas, P.; Black, D. S.; Breen, J.; Collins, T.; Memoli, S.; Miyamoto, J.; Polyakoff, M.; Tumas, W.; Pure Appl. Chem. 2000, 72, 1207.

10. Collins T. J. In Macmillan encyclopedia of chemistry, Lagowski. J. J., ed.; Macmillan: 1997, pp. 691-697.

11. Lankey, R. L.; Anastas, P. T.; Ind. Eng. Chem. Res. 2002, 41, 4498.
12. Marques, C. A.; Machado, A. A. S. C.; Found. Chem. 2019, submitted.

13. Eilks, I.; Zuin, V. G.; Curr. Opin. Green Sustain. Chem. 2018, 13, A4.

14. Welton, T.; Curr. Opin. Green Sustain. Chem. 2018, 13, A7.

15. Mahaffy, P. G.; Krief, A.; Hopf, H.; Mehta, G.; Matlin, S. A.; Nat. Rev. Chem. 2018, 2, 0126.

16. Clark, J. H.; Sheldon, R. A.; Raston, C.; Poliakoff, M.; Leitner, W.; Green Chem. 2014, 16, 18.

17. Marques, C. A.; Machado, A. A. S. C.; Found. Chem. 2014, 16, 125.

18. Woodhouse, E. J.; Breyman, S.; Sci. Technol. Hum. Values 2005, 30, 199.

19. Anastas, P. T.; Han, B.; Leitner, W.; Poliakoff, M.; Green Chem. 2016, $18,12$.

20. Clark, J. H.; Farmer, T. J.; Herrero-Davila, L.; Sherwood, J.; Green Chem. 2016, 18, 3914.

21. Marion, P.; Bernela, B.; Piccirilli, A.; Estrine, B.; Patouillard, N.; Guilbot, J.; Jérôme, F.; Green Chem. 2017, 19, 4973.

22. Sheldon, R. A.; Green Chem. 2017, 19, 18.

23. Collins, T. J.; J. Cleaner Prod. 2017, 140, 93.

24. Graedel, T. E.; Pure Appl. Chem. 2001, 73, 1243.

25. Anastas, P. T.; Green Chem. 2003, 5, G29.

26. Anastas, P. T.; Chem. Eng. News Arch. 2011, 89, 62.

27. Morin, E.; Ciência com consciência, Bertrand Brasil: Rio de Janeiro, 2010.

28. Agazzi, E.; El bien, el mal y la ciencia: las dimensiones éticas de la empresa científico-tecnológica, Tecnos: Madrid, 1996.

29. Pidd, M.; Tools for Thinking: Modelling in Management Science, John Willey and Sons: Chichester, 1996.

30. Checkland, P.; Syst. Res. Behav. Sci. 2000, 17, S11.

31. Bellini, C. G. P; Rech, I.; Borenstein, D.; RAE Eletrônica 2004, 3.

32. Leff, E.; Ezcurra, E.; Pisanty, I.; Romero Lankao, P.; La transición hacia el desarrollo sustentable: perspectivas de América Latina y el Caribe, $1^{\text {a }}$ ed., PNUMA: Ciudad de México, 2002.

33. Anastas, P. T.; Breen, J. J.; J. Cleaner Prod. 1997, 5, 97.

34. Graedel, T. E.; Green Chem. 1999, 1, G126.

35. Bunge, M.; Philos. Soc. Sci. 1997, 27, 410.

36. Constable, D. J. C.; Curr. Opin. Green Sustain. Chem. 2017, 7, 60.

37. Orgill, M.; York, S.; MacKellar, J.; J. Chem. Educ. 2019, 96, 2720.

38. Matlin, S. A.; Mehta, G.; Hopf, H.; Krief, A.; Nat. Chem. 2016, 8, 393.

39. United Nations, Resolution adopted by the General Assembly. 60/1. 2005 World Summit Outcome, UN: New York, 2005.

40. Ribeiro, J. A. G.; Cavassan, O.; Góndola, Enseñanza y Aprendizaje de las Ciencias 2013, 8, 61.

41. Anastas P. T., Levy I. J., Parent K. E.; Green chemistry education: changing the course of chemistry, Oxford University Press: New York, 2009.

42. Marques, C. A.; Machado, A. A. S. C.; REEC Revista Electrónica de Enseñanza de las Ciencias 2018, 17, 19.

43. Vilches, A.; Gil-Pérez, D.; Educ. Quím. 2013, 24, 199.

44. Costa, D. A.; Ribeiro, M. G. T. C.; Machado, A. A. S. C.; Bol. Soc. Port. Quím. 2008, 109, 47.

45. Machado A. A. S. C. In Worldwide Trends in Green Chemistry Education, Zuin V. G., Mammino, L., eds.; Royal Society of Chemistry: London, 2015, pp. 111-136.

46. Burmeister, M.; Rauch, F.; Eilks, I.; Chem. Educ. Res. Pract. 2012, 13, 59.

47. UNESCO; United Nations Decade of Education for Sustainable Development (2005-2014): international implementation scheme, UNESCO: Paris, 2005.

48. Sjöström, J.; Eilks, I.; Zuin, V. G.; Sci. Educ. 2016, 25, 321.

49. Haack, J. A.; Hutchison, J. E.; ACS Sustainable Chem. Eng. 2016, 4, 5889.

50. Płotka-Wasylka, J.; Kurowska-Susdorf, A.; Sajid, M.; de la Guardia, M.; Namieśnik, J.; Tobiszewski, M.; ChemSusChem 2018, 11, 2845. 
51. Goes, L. F. de; Leal, S. H.; Cono, P.; Fernandez, C.; Educ. Quím. 2013, $24,113$.

52. Zuin V. G. and Marques C. A. In Science Education Research and Education for Sustainable Development (ESD); Eilks, I., Markic, S., Ralle, B., eds; Shaker Verlag: Aachen, 2014, pp. 21-41.

53. Duarte, R.; Ribeiro, M. G.; Machado, A.; Bol. Soc. Port. Quím. 2015, $138,36$.

54. Mahaffy, P. G.; Ho, F. M.; Haack, J. A.; Brush, E. J.; J. Chem. Educ. 2019, 96, 2679.

55. Aubrecht, K. B.; Dori, Y. J.; Holme, T. A.; Lavi, R.; Matlin, S. A.; Orgill, M.; Skaza-Acosta, H.; J. Chem. Educ. 2019, 96, 2888.

56. York, S.; Lavi, R.; Dori, Y. J.; Orgill, M.; J. Chem. Educ. 2019, 96, 2742.

57. Pazicni, S.; Flynn, A. B.; J. Chem. Educ. 2019, 96, 2752.

58. Hurst, G. A.; Slootweg, J. C.; Balu, A. M.; Climent-Bellido, M. S.; Gomera, A.; Gomez, P.; Luque, R.; Mammino, L.; Spanevello, R. A.; Saito, K.; Ibanez, J. G.; J. Chem. Educ. 2019, 96, 2794.

59. Mammino, L.; J. Chem. Educ. 2019, 96, 2881.

60. Miller, J. L.; Wentzel, M. T.; Clark, J. H.; Hurst, G. A.; J. Chem. Educ. 2019, 96, 3006.

61. Perosa, A.; Gonella, F.; Spagnolo, S.; J. Chem. Educ. 2019, 96, 2784

62. Odum, H. T.; Environmental Accounting, Emergy and Environmental Decision Making; Wiley: New York, 1996.

63. Mahaffy, P. G.; Ho, F. M.; Haack, J. A.; Brush, E. J.; J. Chem. Educ. 2019, 96, 2679.

64. Richmond, B.; Syst. Dynam. Rev. 1993, 9, 113.

65. Murphy, K. C.; Dilip, M.; Quattrucci, J. G.; Mitroka, S. M.; Andreatta, J. R.; J. Chem. Educ. 2019, 96, 2993.

66. https://iupac.org/systems-thinking-and-green-and-sustainablechemistry/, accessed in July 2020.

67. http://www.iocd.org/Systems/intro.shtml, accessed in July 2020.

68. Mahaffy, P. G.; Matlin, S. A.; Whalen, J. M.; Holme, T. A.; J. Chem. Educ. 2019, 96, 2730.

69. Eaton, A. C.; Delaney, S.; Schultz, M.; J. Chem. Educ. 2019, 96, 2968.

70. Lasker, G. A.; Simcox, N. J.; Mellor, K. E.; Mullins, M. L.; Nesmith, S. M.; van Bergen, S.; Anastas, P. T.; J. Chem. Educ. 2019, 96, 720.

71. Josephson, P.; Nykvist, V.; Qasim, W.; Blomkvist, B.; Dinér, P.; J. Chem. Educ. 2019, 96, 1389.
72. Eissen, M.; Bahadir, M.; König, B.; Ranke, J.; J. Chem. Educ. 2008, 85, 1000.

73. Stark, A.; Ott, D.; Kralisch, D.; Kreisel, G.; Ondruschka, B.; J. Chem. Educ. 2010, 87, 196.

74. Ribeiro, M. G. T. C.; Machado, A. A. S. C.; J. Chem. Educ. 2011, 88, 947.

75. Ribeiro, M. G. T. C.; Machado, A. A. S. C.; J. Chem. Educ. 2013, 90, 432.

76. Sjöström, J.; Talanquer, V.; J. Chem. Educ. 2014, 91, 1125.

77. Guron, M.; Paul, J. J.; Roeder, M. H.; J. Chem. Educ. 2016, 93, 639.

78. Mahaffy, P. G.; Holme, T. A.; Martin-Visscher, L.; Martin, B. E.; Versprille, A.; Kirchhoff, M.; McKenzie, L.; Towns, M.; J. Chem. Educ. 2017, 94, 1027.

79. Lam, C. H.; Escande, V.; Mellor, K. E.; Zimmerman, J. B.; Anastas, P. T.; J. Chem. Educ. 2019, 96, 761.

80. Mahaffy, P. G.; Matlin, S. A.; Holme, T. A.; MacKellar, J.; Nat. Sustainability 2019, 2, 362.

81. Zuin, V. G.; A Inserção da Dimensão Ambiental na Formação de Professores de Química, 1ª ed., Átomo: Campinas, São Paulo, 2011.

82. Blum, C.; Bunke, D.; Hungsberg, M.; Roelofs, E.; Joas, A.; Joas, R.; Blepp, M.; Stolzenberg, H. C.; Sustain. Chem. Pharm. 2017, 5, 94.

83. Anastas, P. T.; Zimmerman, J. B.; Curr. Opin. Green Sustain. Chem. 2018, 13, 150.

84. Sjöström, J.; Talanquer, V.; Curr. Opin. Green Sustain. Chem. 2018, 13, 16.

85. Sjöström, J.; Int. J. Philos. Chem. 2007, 13, 83.

86. Winterton, N.; Clean Technol. Environ. Policy 2003, 5, 8.

87. Georgescu-Roegen, N.; The Entropy Law and the Economic Process, Harvard University Press: Lincoln, USA, 1971.

88. Huesemann, M. H.; Clean Technol. Environ. Policy 2003, 5, 21.

89. Thornton, J.; Int. J. Occup. Environ. Health 2000, 6, 318.

90. Nóvoa, A.; Profissão professor, 01-2014 ed., Porto Editora: Porto, Portugal, 1999.

91. Zabala, A.; Rosa, E. F. da F.; Farenzena, N.; A prática educativa: como ensinar, ARTMED: Porto Alegre, 2007. 